\title{
Central Executive and Default Mode Network Intranet work Functional Connectivity Patterns in Chronic Migraine
}

X Michelle Androulakis ${ }^{1,2^{\star}}$, Kaitlin A Krebs ${ }^{1}$, Charmaine Jenkins ${ }^{1}$, Nasim Maleki ${ }^{3}$, Alan G Finkel ${ }^{4}$, Chris Rorden $^{5}$ and Roger Newman ${ }^{5}$

${ }^{1}$ Division of Neurology, WJB Dorn VA Medical Center, Columbia, SC, USA

${ }^{2}$ Department of Neurology, University of South Carolina, Columbia, SC, USA

${ }^{3}$ Harvard Medical School, Boston, MA, USA

${ }^{4}$ Carolina Headache Institute, Durham, NC, USA

${ }^{5}$ Department of Psychology, University of South Carolina, Columbia, SC, USA

\begin{abstract}
Background: The neural mechanisms of chronic migraine remain largely unknown but linked to the decreased connectivity to intrinsic brain networks.
\end{abstract}

Objective: To characterize the intranetwork functional connectivity within the Central Executive Network (CEN) and Default Mode Network (DMN) in chronic migraine (CM), with and without medication overuse headache $(\mathrm{MOH})$.

Methods: Using functional magnetic resonance imaging, we performed post-hoc analysis of a total of 136 pairs of nodes to node functional connectivity (NTNC) within the CEN and 6 pairs of NTNC within the DMN in CM ( $\mathrm{n}=13)$ and $\mathrm{CM}^{\mathrm{MOH}}(\mathrm{n}=16)$ as compared to controls, and between these two subgroups.

Results: Connectivity between right ventrolateral prefrontal cortex (PFC) to contralateral anterior thalamus and connectivity between left dorsal PFC/frontal eye field (FEF) to dorsomedial PFC were decreased within the CEN in both $\mathrm{CM}$ and $\mathrm{CM}^{\mathrm{MOH}}$ subgroups. In the $\mathrm{CEN}$, there was more widespread disruption in the $\mathrm{CM}^{\mathrm{MOH}}(\mathrm{n}=16)$ versus $\mathrm{CM}$ $(\mathrm{n}=13)$, when compared to healthy controls. Within the subgroups, connectivity between right inferior frontal gyrus to left dorsolateral PFC was decreased in $\mathrm{CM}^{\mathrm{MOH}}$ compared to CM. In the DMN, only one NTNC (left lateral parietal to precuneus/PCC) was disrupted in the $\mathrm{CM}^{\mathrm{MOH}}$ group when compared to controls.

Conclusion: There are similar patterns of NTNC dysfunction within CEN in CM regardless of MOH status. We observed more extensive intranetwork disruption in $\mathrm{CM}^{\mathrm{MOH}}$ than $\mathrm{CM}$. The decreased coherence between the right inferior frontal gyrus and the left dorsolateral $\mathrm{PFC}$ in $\mathrm{CM}^{\mathrm{MOH}}$ is likely associated with a significant disruption in the inhibitory control and a maladaptive response in risk aversion and reward; whereas the decreased coherence between right dorsolateral and ventrolateral PFC to contralateral dorsal PFC/FEF may be related to lack of cognitive control and top-down regulation of pain in both $\mathrm{CM}$ and $\mathrm{CM}^{\mathrm{MOH}}$.

Keywords: Chronic migraine; Central executive network; Default mode network; Medication overuse headache; Functional MRI

\section{Introduction}

Neuroimaging studies have significantly advanced our knowledge on the functional and structural changes of the cortical and subcortical regions in chronic migraine (CM) [1]. Recently, studies have utilized intrinsic resting state functional connectivity networks as a tool for analyzing functional brain alterations in various neurological disorders. Alteration in intrinsic Functional Brain Networks (IFBNs), such as the default mode network (DMN), central executive network (CEN) and salience network (SN) may serve as neuroimaging biomarkers for evaluating the pathophysiological consequence of repeated migraine attacks. These IFBNs are comprised of brain regions that are functionally linked at rest when the subject is not involved in performing any externally-oriented task. The DMN is one of the most consistently described IFBNs and has been implicated in the regulation of internally-oriented processes, including self-monitoring and autobiographical functions [2]. The CEN is involved in goal-directed behavior and is associated with higher-order cognitive processes, such as attention and working memory. The CEN executes control in response to salient input during the selection of an appropriate action in a fluctuating environmental and self-regulating state [3]. Migraine pain is a complex, multidimensional experience which consists not only of neurobiological, but psychosocial and behavioral domains. When migraine pain becomes more persistent as in CM, brain circuits engaged in pain processing are over-sensitized under the influence of biopsychosocial and behavior influence. Better understanding of the intranetwork connectivity based on functional correlations among different brain regions may shed light on the pathogenesis of CM and reveal potential therapeutic target(s) in improving brain networks synchrony.

Several fMRI studies in episodic migraine (EM) have revealed abnormal connectivity within the CEN and DMN, as well as between the two networks [4]. However, our current understanding on how the

*Corresponding author: X Michelle Androulakis, Department of Neurology, University of South Carolina, 6439 Garners Ferry Rd, Columbia, SC, USA, Tel: 803-776-4000; Fax: 803-545-6051; E-mail: michelle.androulakis@uscmed.sc.edu

Received September 24, 2018; Accepted October 15, 2018; Published October 17, 2018

Citation: Androulakis XM, Krebs KA, Jenkins C, Maleki N, Finkel AG, et al. (2018) Central Executive and Default Mode Network Intranet work Functional Connectivity Patterns in Chronic Migraine. J Neurol Disord 6: 393. doi:10.4172/23296895.1000393

Copyright: ( 2018 Androulakis XM, et al. This is an open-access article distributed under the terms of the Creative Commons Attribution License, which permits unrestricted use, distribution, and reproduction in any medium, provided the original author and source are credited. 
IFBNs are affected in CM continues to be limited. In CM, the processing of migraine pain likely shifts from nociceptive brain regions to other networks important in cognitive, affective, and emotional processing. Currently, the differences and similarities are poorly understood between patients with $\mathrm{CM}$ and those with $\mathrm{CM}$ who overuse acute pain medication. Availability of such knowledge would have significant consequential implications for comprehensive classification of $\mathrm{CM}$ and has proven to be a commonly debated subject [5].

Our recent results demonstrated that for patients with $\mathrm{CM}$ and $\mathrm{CM}^{\mathrm{MOH}}$, the overall connectivity of DMN and CEN was less coherent when compared to controls. To further unravel the functional architecture of CM, we propose to examine the NTNC changes within the CEN and DMN in CM and $\mathrm{CM}^{\mathrm{MOH}}$ in this post-hoc analysis. As there are many unique NTNC pairs within each of these networks, further examination of these connections within each network can provide additional information not revealed by the overall or regional "node" resting-state functional connectivity in a network (which averages of all connections to one node). Most importantly, we hope to identify potential cortical targets for neuromodulation such as transcranial direct current stimulation (tDCS) in CM. In tDCS, a low amplitude direct current $(1-2 \mathrm{~mA})$ is delivered; the current modulates intrinsic neuronal activity by altering the resting membrane potential [6] and improves the connectivity of large-scale brain networks via increased synchronization [7]. We hypothesized that there would be similar patterns of dysfunctional NTNC in both CM groups when compared to controls, however a wider disruption would be present in $\mathrm{CM}^{\mathrm{MOH}}$ as compared to CM. To the best of our knowledge, there is no study investigating CEN and DMN intranetwork connectivity between $\mathrm{CM}^{\mathrm{MOH}}$ and $\mathrm{CM}$.

\section{Methodology}

\section{Participants}

Migraine patients were eligible for the study if they were 18 years or older, met diagnostic criteria outlined in the International Classification of Headache Disorders III beta guidelines (ICDH III beta) [8] for CM as determined by a headache specialist, or were non-pain, non-headache healthy controls. CM subgroups (with or without medication overuse) were further characterized based on ICHD III beta diagnostic criteria. Patients with a history of episodic migraine (EM) who subsequently developed $\mathrm{CM}$ due to excessive use of acute pain medications were included in $\mathrm{CM} \mathrm{MOH}$ subgroup. Participants were excluded if they exhibited the following: any MRI contraindication, neurological or pain disorders other than CM, any chronic illness (i.e. hypertension, diabetes, hepatic, renal, chronic inflammatory, or infectious disease), or inability to follow study protocol while completing assessments. Healthy controls were excluded if they used over the counter/prescription pain medication for more than 5 days per month or if they had a family history of migraine. CM patients were scanned at their baseline level of pain and at least 24 hours outside of their acute pain exacerbation period; any patient who came in within 24 hours of acute pain exacerbation was rescheduled. All participants underwent vital sign and BMI evaluation; additionally, each patient underwent a neurological examination and completed a standardized questionnaire to ascertain demographics including age, sex, race, educational level, and clinical characteristics (listed in Table 1). In our cohort, CM patients had very few headache free days $(<10$ days/month), therefore we used moderate to severe headache days per month as one of a clinical variable in our analysis.

\section{Questionnaires}

The Headache Impact Test (HIT-6) is a validated questionnaire that consists of six items reflecting quality of life measures affected by headache [8]. Higher scores (range of 36-78) indicate an increasing impact of headaches on daily functioning [8]. The Patient Health Questionnaire (PHQ-9) is a diagnostic measure for clinical depression [9]. A score of $\geq 15$ on the PHQ-9 is associated with a $68 \%$ sensitivity and $95 \%$ specificity for diagnosing "major depressive disorder" based on the DSM-IV criteria [9-11].

\section{Standard protocol approvals, registrations, and patient con- sents}

The institutional review board of the University of South Carolina approved the study protocol and written informed consent was collected from all study participants.

\section{MR imaging}

All participants were scanned on a Siemens 3T scanner located at the McCausland Center for Brain Imaging at Palmetto Health Richland Hospital (Columbia, South Carolina). Participants were instructed to keep their eyes closed, stay awake, relax, and think of "nothing in particular" during the resting state scan. Briefly, all imaging parameters are the same as describe in our early study. The functional images were acquired using a T2 weighted BOLD (blood oxygenation dependent) contrast sensitive sequence TR/TE $=1550 / 34$ $\mathrm{ms}, 42$ slices, flip angle $=71^{\circ}$, voxel size $=2.5 \mathrm{~mm}^{3}, \mathrm{FOV}=215 \mathrm{~mm}^{2}$, and using a transversal, descending, interleaved acquisition. Resting-state fMRI (Rs-fMRI) preprocessing was completed using a combination of Statistical Parametric Mapping 12 software and custom Matlab scripts. The pipeline consisted of standard procedures including motion correction, co-registration, normalization, frequency filtering $(0.01$ to $0.1 \mathrm{~Hz}$ band-pass), and spatial smoothing $(8 \mathrm{~mm}$ full width at half maximum). For each network, a connectivity atlas was constructed using spherical (15 mm diameter) regions of interest (ROIs) centered on the peak Montreal Neurological Institute (MNI) coordinates for the a priori networks of the CEN and DMN.

\section{Functional brain networks}

The CEN (illustrated in Figure 1) consists of the bilateral anterior thalamus, bilateral dorsolateral prefrontal cortex (dlPFC), bilateral dorsal PFC region of frontal eye fields (dPFC/FEF), bilateral ventrolateral PFC (vlPFC), dorsal medial PFC (dmPFC), right inferior frontal gyrus (IFG)/frontal operculum, right inferior temporal, bilateral lateral parietal, left orbital-frontal insula (OFI), bilateral dorsal caudate (dCaudate), and right ventromedial caudate (vmCaudate). The DMN (illustrated in Figure 2) consists of the bilateral lateral parietal (LP) regions as well as the medial prefrontal cortex (mPFC), and posterior cingulate cortex (PCC)/Precuneus. The ROIs (nodes) and the corresponding $\mathrm{MNI}$ coordinates used in each network are listed in our previous publication and are illustrated in Figures 1 and 2.

\section{Statistical analysis}

Using the generated ROIs ("nodes"), functional connectivity matrices were created by first extracting the mean value over time from the BOLD signal time series for each node in each network; the Pearson correlation $(r)$ coefficients were then calculated between each pair of nodes within a network. In all networks, Pearson correlation coefficient values were Fischer $\mathrm{Z}$ transformed (to convert to a normal distribution) to produce the "functional connectivity strengths" that are used in the analysis (Figures 3-5). The intranetwork NTNC (also referred to as "edge") strength was defined as the correlation coefficient generated between each pair of ROIs in each network investigated. Comparison groups were formed to investigate the relationship between the following: $\mathrm{CM}^{\mathrm{MOH}}$ vs. controls, $\mathrm{CM}$ vs. controls, and $\mathrm{CM}^{\mathrm{MOH}}$ vs. CM. 
Citation: Androulakis XM, Krebs KA, Jenkins C, Maleki N, Finkel AG, et al. (2018) Central Executive and Default Mode Network Intranet work Functional Connectivity Patterns in Chronic Migraine. J Neurol Disord 6: 393. doi:10.4172/2329-6895.1000393
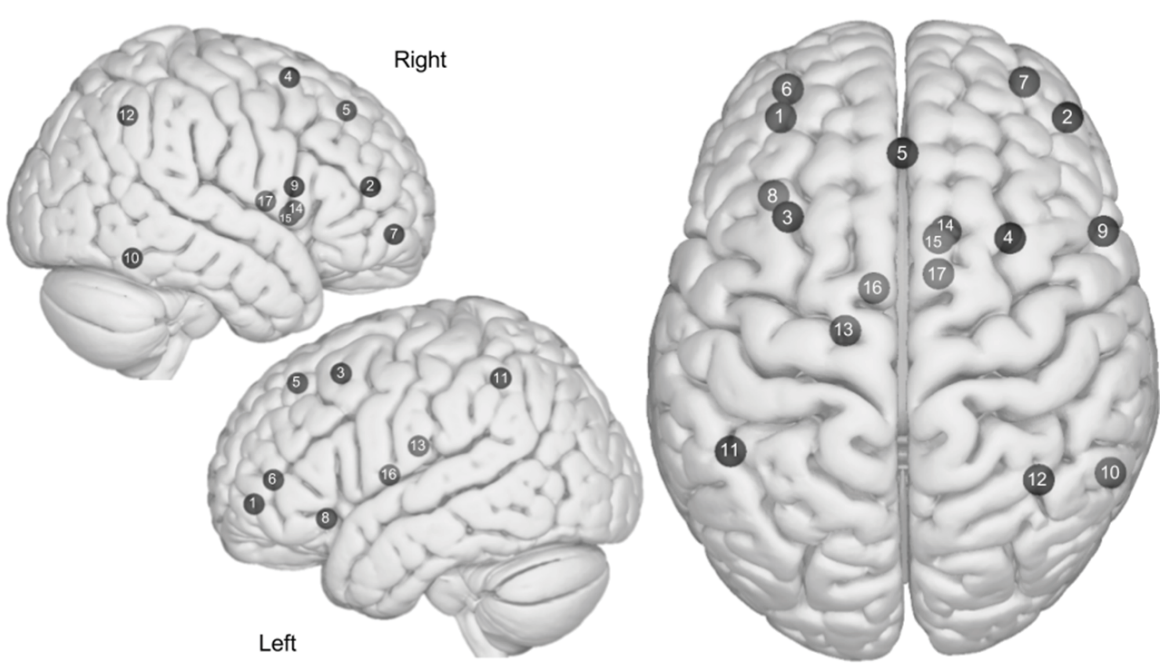

Figure 1: Axial and sagittal view of the Central Executive Network (CEN). (1) Left dIPFC, (2) Right dIPFC, (3) Left dPFC/FEF, (4) Right dPFC/FEF, (5) dm PFC, (6) Left vIPFC, (7) Right vIPFC, (8) Left OFI, (9) Right IFG, (10) Right Inferior Temporal, (11) Left Lateral Parietal, (12) Right Lateral Parietal, (13) Left dCaudate, (14) Right dCaudate, (15) Right vmCaudate, (16) Left Anterior Thalamus, (17) Right anterior thalamus. Images were made with "Surf Ice" (https://www.nitrc.org/projects/ surfice/) using the exact MNI coordinate locations. Node depth is illustrated by transparency.
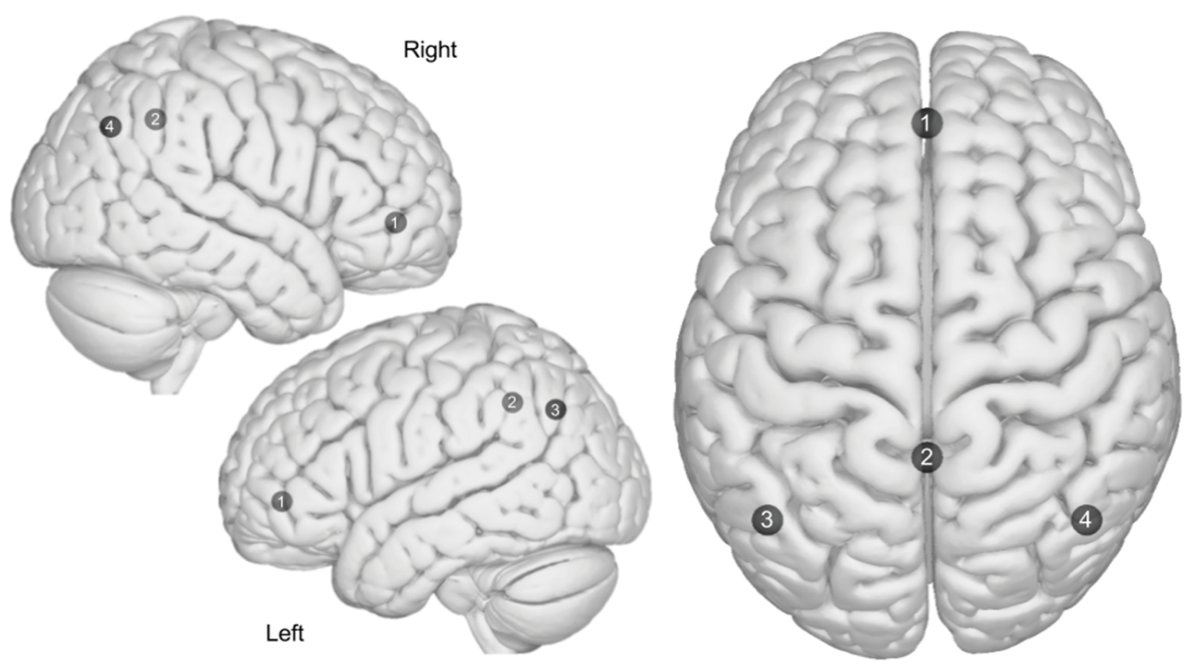

Figure 2: Axial and sagittal view of the Default Mode Network (DMN). (1) Medial prefrontal, (2) Precuneus/PCC, (3) Left lateral parietal, (4) Right lateral parietal. Images were made with "Surf Ice" (https://www.nitrc.org/projects/surfice/) using the exact MNI coordinate locations and sphere sizes. Node depth is illustrated by transparency.

When comparing each subgroup to their matched controls, we used a one tailed, two sample t-test. When comparing between the CM subgroups $\left(\mathrm{CM}^{\mathrm{MOH}}\right.$ vs. $\left.\mathrm{CM}\right)$, a two tailed, two-sample t-test was utilized. A one-tailed t-test was used for $\mathrm{CM} v$ s. controls due to the hypothesis that functional connectivity strengths observed in CM patients would be lower than that of the controls; conversely, a two-tailed t-test was more appropriate when comparing between the two CM subgroups as the hypothesis predicted differences between the groups rather than a change in a specific direction (i.e. an increase or decrease).

To control for multiple comparisons, each statistical test employed a permutation method using 10,000 permutations (MATLAB) [12]. For each of the 9,999 random permutations as well as the one actual ordering, we logged the peak $\mathrm{t}$-score for all regions. We then sorted these maximums and used the $500^{\text {th }}$ most significant peak as our threshold to provide robust control for familywise error. These corrected $p$ values of $<0.05$ were considered significant for intranetwork NTNC strength differences $\left(\mathrm{CM}^{\mathrm{MOH}}\right.$ vs. $\left.\mathrm{CM}\right)$, and the corrected $\mathrm{p}$ values of $<0.01$ were considered significant for intranetwork NTNC strength differences (CM subgroups $v s$. controls).

To assess associations between intranetwork functional connectivity strength and clinical parameters, Pearson correlations were conducted between the shared significant intranetwork NTNC strengths and clinical parameters; these included years with migraine, years with $\mathrm{CM}$, number of moderate to severe headache per month, PHQ-9, HIT-6, and Allodynia symptoms checklist scores (ASC 12). Correlations were corrected for multiple comparison using the Bonferroni correction $(p=0.00015)$. 
Citation: Androulakis XM, Krebs KA, Jenkins C, Maleki N, Finkel AG, et al. (2018) Central Executive and Default Mode Network Intranet work Functional Connectivity Patterns in Chronic Migraine. J Neurol Disord 6: 393. doi:10.4172/2329-6895.1000393

Page 4 of 8

\section{Results}

\section{Participants}

A total of $16 \mathrm{CM}$ and $13 \mathrm{CM}^{\mathrm{MOH}}$ patients were recruited between January 2015 and August 2016 from the headache clinic at University of South Carolina (Columbia, SC). All CM participants were female and age-matched to healthy female controls. We excluded four patients during data analysis due to motion artifact $(\mathrm{n}=1)$, incidental finding on the anatomical T1 image $(n=2)$, and the presence of non-migraine related pain (development of a stomach ulcer) during the scan $(n=1)$. Among the controls $(n=23)$, four were excluded for: claustrophobia $(n=2)$, motion artifact $(\mathrm{n}=1)$, and an incidental finding on the anatomical T1 image $(\mathrm{n}=1)$. Individual characteristics for each group are summarized in Table 1. Of the CM participants, 16 had the secondary diagnosis of $\mathrm{MOH}$ due to overuse of opioids $(n=8)$, triptans $(n=9)$, combined analgesics $(\mathrm{n}=14)$, and combination of triptans with opioids or NSAIDS $(\mathrm{n}=10)$. Among $29 \mathrm{CM}$ participants, 15 used daily migraine preventive prophylaxis medications (topiramate or propranolol). The same cohort was also used in the analysis of Salience Network [13].

\section{CEN}

In the CEN, there was more widespread disruption in the $\mathrm{CM}^{\mathrm{MOH}}$ $(n=16)$ versus CM $(n=13)$, and when compared to healthy controls. Significant intranetwork rs-fc within the CEN when comparing each CM subgroup to controls are listed in Table 2 and illustrated in Figure 3. Significant intranetwork connections when comparing between $\mathrm{CM}$ subgroup are listed in Table 3 and illustrated in Figure 4.

\section{DMN}

The intra-network connection in the DMN (left lateral parietal to precuneus/PCC, $p=0.003$ ) was lower in $\mathrm{CM}$ without $\mathrm{MOH}$ when compared to the matched controls. There was no significant intranetwork connections difference when comparing $\mathrm{CM}^{\mathrm{MOH}}$ and $\mathrm{CM}$.

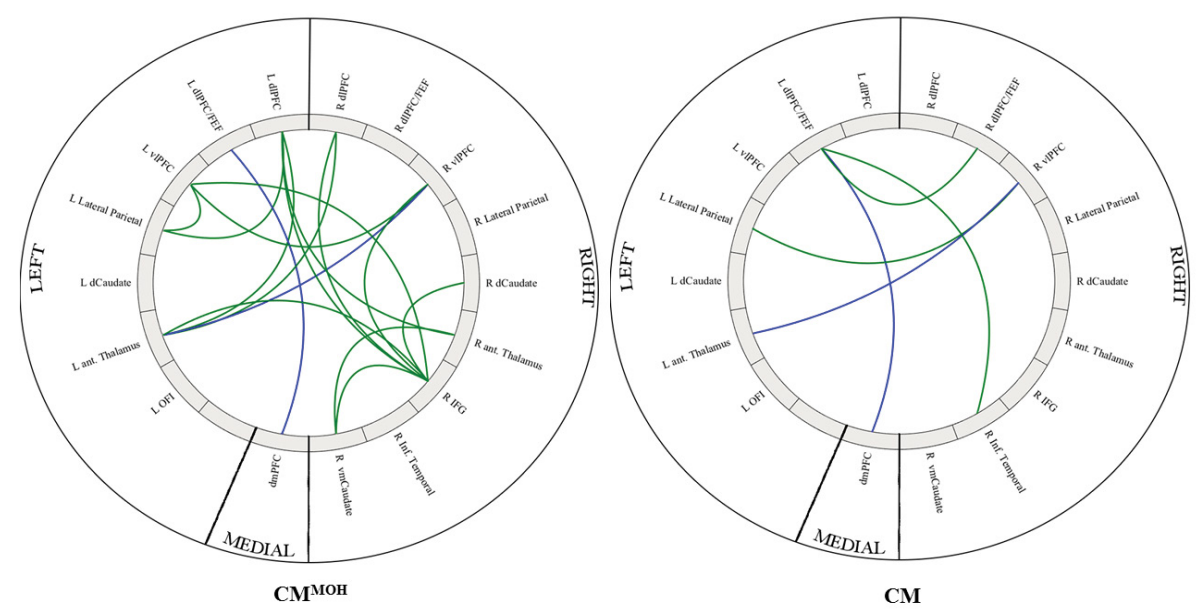

Figure 3: Intra-network connectivity differences in $\mathrm{CM}^{\mathrm{MOH}}$ as compared to controls (left) and $\mathrm{CM}$ as compared to controls (right). Colored lines represents a significant intra-network connectivity difference between the CM groups as compared to matched controls. Green represents decreased connectivity as compared to controls, and blue represents shared decreased difference in both subgroups. Images were generated using the Matlab application "Circro" (https://github.com/ bonilhamusclab/circro).

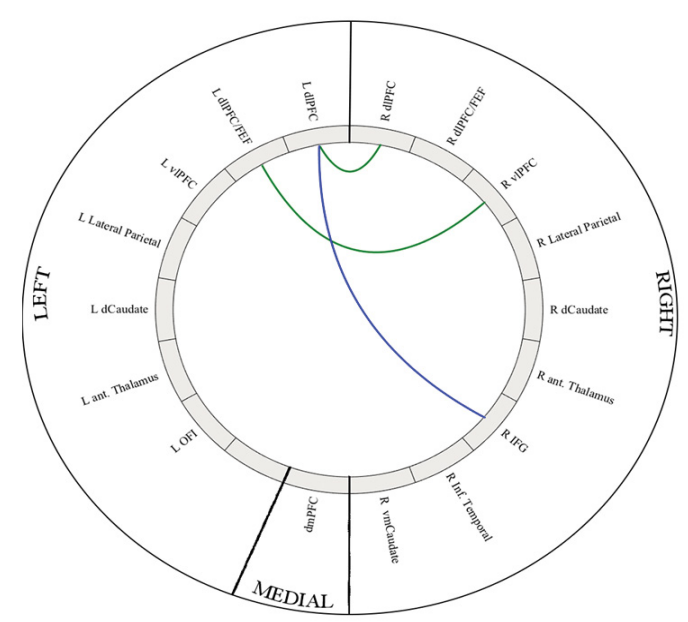

Figure 4: Colored lines represent intra-network connectivity differences between the $\mathrm{CM}^{\mathrm{moH}}$ and $\mathrm{CM}$. Green represents the intra-network connection that was lower in CM patients, blue represents the intra-network connection that was lower in $\mathrm{CM}^{\mathrm{MOH}}$ patients. Images were generated using the Matlab application "Circro" (https:// github.com/bonilhamusclab/circro). 
Citation: Androulakis XM, Krebs KA, Jenkins C, Maleki N, Finkel AG, et al. (2018) Central Executive and Default Mode Network Intranet work Functional Connectivity Patterns in Chronic Migraine. J Neurol Disord 6: 393. doi:10.4172/2329-6895.1000393

Page 5 of 8

\begin{tabular}{|c|c|c|c|}
\hline Demographics & СMMOH (16) & CM (13) & Controls (19) \\
\hline Age & $39 \pm 14$ & $39 \pm 12$ & $37 \pm 11$ \\
\hline BMI & $26 \pm 6$ & $31 \pm 4$ & $26 \pm 5$ \\
\hline \multicolumn{4}{|c|}{ Race/Ethnicity } \\
\hline white & $10(62.5)$ & $10(76.9)$ & $12(63.1)$ \\
\hline black & $6(37.5)$ & $3(23.1)$ & $7(36.9)$ \\
\hline \multicolumn{4}{|c|}{ Highest Degree Completed } \\
\hline Unknown & $1(6.3)$ & $1(7.7)$ & $1(5.3)$ \\
\hline High School & $5(31.3)$ & $8(61.5)$ & $4(21)$ \\
\hline Undergraduate & $7(43.8)$ & $2(15.4)$ & $10(52.6)$ \\
\hline Graduate & $3(18.8)$ & $2(15.4)$ & $4(21.0)$ \\
\hline Clinical Features & СMMOH & CM & $p$-value \\
\hline Moderate to severe HA days/month & $21 \pm 7$ & $15 \pm 5$ & $0.005^{\star}$ \\
\hline \multicolumn{4}{|c|}{ Headache Location } \\
\hline Right & $1(6.3)$ & $2(15.4)$ & $\mathrm{n} / \mathrm{a}$ \\
\hline Left & $2(12.5)$ & $3(23.1)$ & -- \\
\hline Bilateral & $13(81.3)$ & $8(61.5)$ & -- \\
\hline Aura & $10(62.5)$ & $5(38.5)$ & $\mathrm{n} / \mathrm{a}$ \\
\hline Cranial autonomic symptoms & $8(5 \mathrm{~h} 0.0)$ & $2(15.4)$ & $\mathrm{n} / \mathrm{a}$ \\
\hline Allodynia (ASC12) Score & $6 \pm 3$ & $5 \pm 6$ & 0.22 \\
\hline Family history ( $1^{\text {st }}$ degree) & $13(81.3)$ & $7(53.8)$ & $\mathrm{n} / \mathrm{a}$ \\
\hline History of migraine (years) & $21 \pm 13$ & $20 \pm 13$ & 0.371 \\
\hline History of CM (years) & $2 \pm 1$ & $3 \pm 3$ & 0.135 \\
\hline Hit-6 Score & $67 \pm 3$ & $64 \pm 4$ & $0.03^{*}$ \\
\hline PHQ-9 Score & $10 \pm 6$ & $5 \pm 3$ & $0.006^{*}$ \\
\hline
\end{tabular}

Abbreviations: $\mathrm{CM}=$ Chronic Migraine; $\mathrm{MOH}=$ Medication Overuse Headache; $\mathrm{c}=$ controls; BMI=Body Mass Index; HA=Moderate to Severe Headache Days; HIT6=Headache Impact Test; PHQ-9=Patient Health Questionnaire-9

Values represent mean \pm standard deviation or number ( $\%$ of total).

Table 1: Clinical characteristics of participants.

\begin{tabular}{|c|c|c|c|}
\hline \multicolumn{4}{|c|}{ Node to Node connection } \\
\hline CEN: Decreased coherence in $\mathrm{CM}^{\text {мон }}$ & $\mathrm{CM}^{\text {Mон }}$ & Control & $p$-value \\
\hline Left Anterior Thalamus $\times$ right vIPFC ${ }^{*}$ & $0.13 \pm 0.14$ & $0.25 \pm 0.14$ & 0.008 \\
\hline Left Anterior Thalamus $\times$ right dIPFC & $0.07 \pm 0.17$ & $0.29 \pm 0.16$ & $<0.001$ \\
\hline Left dIPFC $\times$ left Anterior Thalamus & $0.16 \pm 0.13$ & $0.32 \pm 0.14$ & 0.001 \\
\hline Left dIPFC $\times$ right Anterior Thalamus & $0.14 \pm 0.12$ & $0.27 \pm 0.16$ & 0.007 \\
\hline Left dPFC/FEF $\times$ dmPFC ${ }^{\star}$ & $0.53 \pm 0.31$ & $0.79 \pm 0.19$ & 0.006 \\
\hline Left dIPFC $\times$ left Lateral Parietal & $0.34 \pm 0.24$ & $0.61 \pm 0.28$ & 0.004 \\
\hline Right IFG $\times$ left dIPFC & $0.08 \pm 0.15$ & $0.37 \pm 0.19$ & $<0.001$ \\
\hline Right IFG $\times$ right dIPFC & $0.39 \pm 0.24$ & $0.64 \pm 0.16$ & $<0.001$ \\
\hline Right IFG $\times$ left vIPFC & $0.05 \pm 0.19$ & $0.24 \pm 0.17$ & 0.005 \\
\hline Right IFG $\times$ right vIPFC & $0.16 \pm 0.18$ & $0.30 \pm 0.14$ & 0.003 \\
\hline Right IFG $\times$ right vmCaudate & $0.03 \pm 0.23$ & $0.24 \pm 0.10$ & 0.001 \\
\hline Right IFG $\times$ right dCaudate & $0.04 \pm 0.20$ & $0.21 \pm 0.17$ & 0.006 \\
\hline Right IFG $\times$ left Anterior Thalamus & $0.11 \pm 0.21$ & $0.38 \pm 0.22$ & 0.002 \\
\hline Right IFG $\times$ right Anterior Thalamus & $0.16 \pm 0.23$ & $0.38 \pm 0.21$ & 0.005 \\
\hline Left vIPFC $\times$ left Lateral Parietal & $0.45 \pm 0.25$ & $0.71 \pm 0.25$ & 0.007 \\
\hline Left vIPFC $\times$ right vIPFC & $0.57 \pm 0.28$ & $0.83 \pm 0.23$ & 0.005 \\
\hline CEN: Decreased coherence in CM & CM & Control & $p$-value \\
\hline Right vIPFC $\times$ left Lateral Parietal & $0.35 \pm 0.19$ & $0.57 \pm 0.22$ & 0.002 \\
\hline Left Anterior Thalamus $\times$ right vIPFC* & $0.10 \pm 0.12$ & $0.25 \pm 0.14$ & 0.008 \\
\hline Left dPFC/FEF $\times$ right Inferior Temporal & $0.19 \pm 0.11$ & $0.30 \pm 0.10$ & 0.004 \\
\hline Left dPFC/FEF $\times$ right dPFC/FEF & $0.22 \pm 0.17$ & $0.43 \pm 0.24$ & 0.0095 \\
\hline Left dPFC/FEF $\times$ dmPFC ${ }^{*}$ & $0.56 \pm 0.23$ & $0.82 \pm 0.19$ & $<0.001$ \\
\hline DMN: Decreased coherence in $\mathrm{CM}^{\mathrm{MOH}}$ & $\mathrm{CM}^{\mathrm{MOH}}$ & Control & $p$-value \\
\hline Left Lateral Parietal $\times$ Precuneus/PCC & $0.75 \pm 0.18$ & $0.49 \pm 0.30$ & 0.003 \\
\hline \multicolumn{4}{|c|}{$\begin{array}{l}\text { DMN: Decreased coherence in CM } \\
\text { None }\end{array}$} \\
\hline
\end{tabular}

Table 2: Significant differences of intranetwork connections in the CEN for each CM subgroup as compared to controls. 


\begin{tabular}{|c|c|c|c|c|}
\hline Variables & Controls & СМмон & CM & $\begin{array}{c}p \text {-value } \\
\left(\mathrm{CM}^{\mathrm{MOH}} \text { vs. CM) }\right.\end{array}$ \\
\hline \multicolumn{5}{|c|}{ Decreased coherence in $\mathrm{CM}^{\mathrm{MOH}}$ as compared to $\mathrm{CM}$} \\
\hline Right IFG $\times$ Left dIPFC ${ }^{*}$ & $0.35 \pm 0.19$ & $0.08 \pm 0.15$ & $0.24 \pm 0.22$ & 0.040 \\
\hline \multicolumn{5}{|c|}{ Decreased coherence in $\mathrm{CM}$ as compared to $\mathrm{CM}^{\mathrm{MOH}}$} \\
\hline Left dPFC/FEF $\times$ Right vIPFC & $0.44 \pm 0.22$ & $0.41 \pm 0.24$ & $0.22 \pm 0.27$ & 0.007 \\
\hline Left dPFC/FEF $\times$ Right dIPFC & $0.10 \pm 0.26$ & $0.21 \pm 0.32$ & $0.00 \pm 0.29$ & 0.043 \\
\hline
\end{tabular}

Table 3: Significant differences ( $p<0.05$; two tailed) of intranetwork functional connectivity $(\mathrm{Avg} \pm \mathrm{SD})$ when comparing $\mathrm{CMMOH}$ vs. CM.

\section{Correlations}

After correcting for multiple comparisons, we found no correlational relationship between clinical features and intranetwork connectivity using Pearson correlation.

\section{Discussion}

\section{Impairment in PFC-mediated descending pain control in $\mathrm{CM}$ and $\mathrm{CM}^{\mathrm{MOH}}$}

In this study, we examined the intranetwork functional connectivity within the CEN and DMN in CM and $\mathrm{CM}^{\mathrm{MOH}}$. Our results indicate decreased connectivity of the PFC within the CEN, regardless of medication overuse status in these patients. These findings support the notion that decreased connectivity between PFC and deep subcortical structures, that are important in controlling/inhibiting pain, may play a significant role in the pathophysiology of CM. For instance, vlPFC is critical in the cognitive modulation of pain, with the ability to initiate the downstream modulation of pain [14]. Increased activation of the vlPFC is associated with analgesic effect [15]. The impaired connections between vlPFC to the thalamus is intriguing, as chronic pain conditions are often reported to be associated with decreased thalamic activity due to an adaptive response to the continuous or recurrent pain and may play an important role in development and maintenance of the chronic pain $[16,17]$. As both CM subgroups are experiencing chronic pain, it is not surprising that we observed the decreased connectivity between the vlPFC and anterior thalamus in both $\mathrm{CM}$ and $\mathrm{CM}^{\mathrm{MOH}}$. In fact, our data demonstrate impaired inhibition of the descending pain control in both subgroups, with a more pronounced effect in $\mathrm{CM}^{\mathrm{MOH}}$ (Table 2).

\section{Enhanced CEN dysfunction in $\mathrm{CM}^{\mathrm{MOH}}$}

In this study, the presence of $\mathrm{MOH}$ was associated with more widespread network dysfunction, as evidenced by the increased number of aberrant NTNC within the CEN. The nodes primarily affected were within the CEN overlapping thalamic systems, PFC, and the inferior frontal gyrus (IFG). These additional NTNC dysfunctions are likely to be related to the dependency to analgesic medication given that the chronic overuse of pain medications contributes to decreased pain threshold and development of addictive/compulsive traits [18].

Many of the affected NTNC in our $\mathrm{CM}^{\mathrm{MOH}}$ cohort involve regions of the cortico-basal ganglia-thalamic system. Specifically, regions that function act as the "associative/cognitive" (frontal lobe-caudatethalamus) component of thalamic systems, which also overlaps with the components (nodes) within the CEN $[19,20]$. Specifically, when compared to healthy controls, widespread dysfunctions were seen in regions, such as the dorsomedial $\mathrm{PFC}$, dlPFC, anterior thalamus, right dorsal, and ventromedial caudate in $\mathrm{CM}^{\mathrm{MOH}}$. The role of chronic overuse of pain medications on the PFC connectivity is supported by metabolic changes in the dlPFC that are associated with risk aversion/reward and compulsive drug seeking behavior [21]. Specifically, on a neural level, it is believed that when goal directed behavior becomes habit formation (via repetitive drug use), "cognitive control" is transitioned from the PFC to striatum [22]. This transition may result in less connectivity of PFC regions due to transfer of cognitive control.

Besides changes in PFC and thalamus connectivity, one of the most prominent results for the $\mathrm{CM}^{\mathrm{MOH}}$ group is the unique pattern of dysfunction observed in the right inferior frontal gyrus (IFG). This decreased connectivity may be associated with the neurological changes from the chronic overuse of pain medication, as the IFG plays a vital role in response inhibition. Response inhibition is the suppression of an action that is either contextually inappropriate or interrupts a goal-oriented behavior and is an important element in executive control $[19,23]$. Response inhibition is initiated by IFG and require the subthalamic nucleus for inhibition of thalamic projections to the cortex $[19,23]$. Our data suggests that the overuse of pain medication may further compromise the CEN in the $\mathrm{CM}^{\mathrm{MOH}}$ due to the dysfunction of the IFG. The notion that chronic overuse of medication further complicates $\mathrm{CM}$ is also supported by the concurrent differences in headache-related clinical parameters found between the two $\mathrm{CM}$ subgroups. We noted a significantly higher number of headache days in $\mathrm{CM}^{\mathrm{MOH}}$, and stronger functional impact of headaches in this cohort as indicated by significantly higher HIT-6 scores (Table 1). These headache characteristics in $\mathrm{MOH}$ group further support our hypothesis that the greater degree of dysfunctional connectivity is associated with phenotypes of $\mathrm{CM}^{\mathrm{MOH}}$.

\section{DMN: No intranet work connectivity differences between $\mathrm{CM}$ and $\mathrm{CM}^{\mathrm{MOH}}$}

We did not observe any NTNC differences when comparing CM and $\mathrm{CM}^{\mathrm{MOH}}$; this is consistent with our previous findings that the overall DMN connectivity is not different between the subgroups. Brain regions within $\mathrm{DMN}$ are likely not key in differentiating $\mathrm{MOH}$ versus non $\mathrm{MOH}$ in $\mathrm{CM}$. Previous reports of decreased connectivity between left lateral parietal and precuneus regions in chronic pain patients, is consistent with our findings for the $\mathrm{MOH}$ group as compared to controls, but not the CM group [24]. This suggests that $\mathrm{CM}^{\mathrm{MOH}}$ patients may have more of a "classic chronic pain" pattern of network disruption, whereas CM patients do not.

\section{Study Limitations}

We acknowledge that there are several limitations with this study. First, medication usage is a confounder that may have influenced the results. Some CM participants $(n=15)$ were on daily migraine prophylaxis medications, but we do not believe this impacted our results based on our previously reported findings [13]. Additionally, differences in the usage of acute pain medications (i.e. type, dosing) may impact the functional connectivity. Secondly, participants were not matched exactly according to anxiety/depression scale; therefore, we 

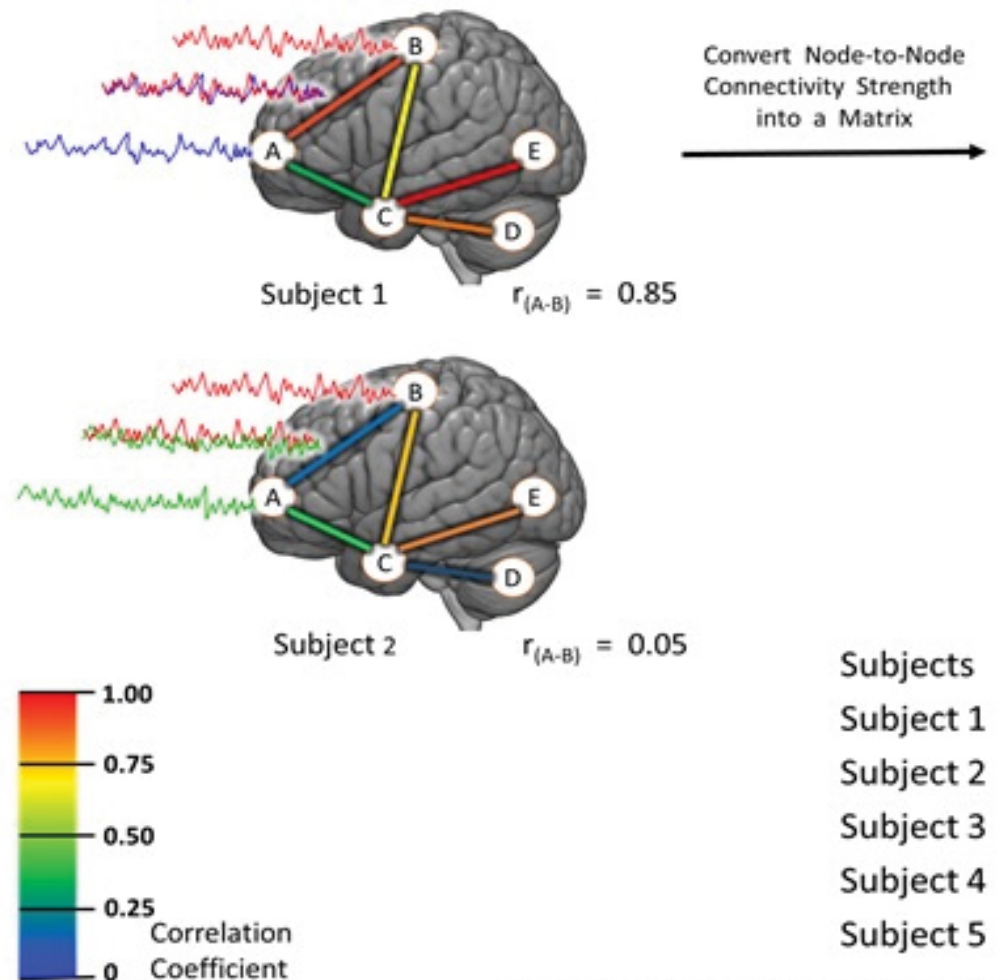

( $r$ ) Scaling

for Node-to-

Node

Connectivity

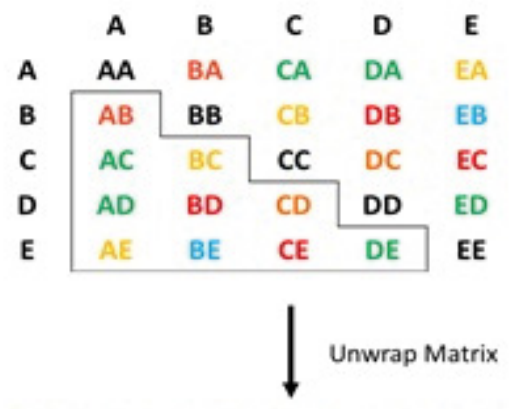

$A B A C B C A D B D C D$ AE BE CE DE Combine all matrix from All Subjects

Subjects Network

Subject 1 AB AC BC AD BD CD AE BE CE DE

Subject $2 \quad A B \mid A C B C$ AD BD CD AE BE CE DE

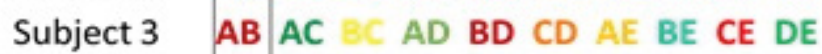

Subject $4 \quad A B \quad A C$ BC AD BD CD AE BE CE DE

Subject $5 \quad A B$ AC BC AD BD CD AE BE CE DE

Average Node-to-Node Connectivity Strength

Figure 5: A schematic diagram of fMRI data processing workflow. After fMRI time series activity level for each individual node is estimated for each participant, then BOLD signals from each node are correlated to other nodes within a priori network, and the $\mathrm{R}$ value (correlation coefficient) is calculated for each pair (edge). A table is then generated for each pair of nodes, containing all edges for each network per participant. Average node to node connectivity is calculated by averaging the $\mathrm{R}$ value of the same edge within the network in all participants.

cannot exclude the possibility that some of the changes in connectivity may be related (cause or effect) to anxiety/depression. Interestingly, we did not find any correlation between NTNC and clinical characteristics. This may be due to our highly conservative statistical approach involving Bonferroni corrections which are known to be associated with higher type II error rate (false negative). One interesting possibility is that genetic or epigenetic differences in $\mathrm{CM}$ and $\mathrm{CM}^{\mathrm{MOH}}$ are better correlated than purely the clinical parameters used, which is subjective to recall bias. Thirdly, the sample size in this study is small and therefore we may not have had sufficient power to detect more subtle effects. Lastly, we cannot ascertain if the changes in connectivity observed are causal or an effect of chronic pain, which is a common limitation in similar studies using cross-sectional study design.

\section{Conclusion}

In this study, we were able to characterize the intranet work functional connectivity patterns within two of the major IFBN: CEN and DMN, as well as examine the influence of medication overuse on the connectivity patterns in the brain. We found that while there are shared dysfunctional NTNC within CEN in CM, regardless of $\mathrm{MOH}$ status, the intranetwork connectivity disruptions were more extensive in $\mathrm{CM}^{\mathrm{MOH}}$. We propose that the dysfunction of the regions within the CEN plays an important role in CM pathogenesis, and that $\mathrm{PFC}$ (dlPFC, vlPFC) and IFG can serve as therapeutic targets to improve CEN synchronization through tDCS.

\section{Clinical Implications}

- Dysfunction of regions within the central executive network (CEN) plays an important role in chronic migraine (CM) pathogenesis.

- While there are shared dysfunctional node-to-node connections within the $\mathrm{CEN}$ in $\mathrm{CM}$ regardless of medication overuse headache $(\mathrm{MOH})$ status, intranetwork connectivity disruptions were more extensive in $\mathrm{CM}^{\mathrm{MOH}}$

- The prefrontal cortex (dlPFC, vlPFC) and inferior frontal gyrus (IFG) may serve as therapeutic targets to improve CEN synchronization through transcranial direct current stimulation.

\section{Acknowledgements}

We would like to thank Dr. Taylor Hanayik (University of South Carolina), for his assistance in analysis and interpretation of data. We also want to thank our patients and healthy control volunteers for their participation. 
Citation: Androulakis XM, Krebs KA, Jenkins C, Maleki N, Finkel AG, et al. (2018) Central Executive and Default Mode Network Intranet work Functional Connectivity Patterns in Chronic Migraine. J Neurol Disord 6: 393. doi:10.4172/2329-6895.1000393

\section{Funding}

This work is supported and funded by National Institutes of Health (NIH) (R21NS099760 (NIH/NINDS)).

\section{References}

1. Schwedt TJ, Chong CD, Wu T, Gaw N, Fu Y, et al. (2015) Accurate classification of chronic migraine via brain magnetic resonance imaging. Headache 55: 762-777.

2. Bressler SL, Menon V (2010) Large-scale brain networks in cognition: Emerging methods and principles. Trends Cogn Sci 14: 277-290.

3. Seeley WW, Menon V, Schatzberg AF, Keller J, Glover GH, et al. (2007) Dissociable intrinsic connectivity networks for salience processing and executive control. J Neurosci 27: 2349-2356

4. Xue T, Yuan K, Zhao L, Dong T, Cheng P, et al. (2012) Intrinsic brain network abnormalities in migraines without aura revealed in resting-state fMRI. PloS one 7: e52927.

5. Olesen J, Bousser MG, Diener HC, Dodick D, First M, et al. (2006) New appendix criteria open for a broader concept of chronic migraine. Cephalalgia 26: 742-746.

6. Stagg CJ, Nitsche MA (2011) Physiological basis of transcranial direct current stimulation. Neuroscientist 17: 37-53.

7. Peña-Gómez C, Sala-Lonch R, Junqué C, Clemente IC, Vidal D, et al. (2012) Modulation of large-scale brain networks by transcranial direct current stimulation evidenced by resting-state functional MRI. Brain Stimul 5: 252-263.

8. Headache Classification Committee of the International Headache Society (IHS) (2013) The international classification of headache disorders, $3^{\text {rd }}$ edition (beta version). Cephalalgia 33: 629-808.

9. Shin HE, Park JW, Kim YI, Lee KS (2008) Headache Impact Test-6 (HIT-6) scores for migraine patients: Their relation to disability as measured from a headache diary. J Clin Neurol 4: 158-163.

10. Seo JG, Park SP (2015) Validation of the Patient Health Questionnaire-9 (PHQ9) and PHQ-2 in patients with migraine. J Headache Pain 16: 65.

11. Freedman D, Lane D (1983) A non-stochastic interpretation of reported significance levels. J Bus Econ Stat 1: 292-298.
12. Winkler AM, Ridgway GR, Webster MA, Smith SM, Nichols TE (2014) Permutation inference for the general linear model. Neuroimage 92: 381-397.

13. Androulakis XM, Rorden C, Peterlin BL, Krebs K (2018) Modulation of salience network intranetwork resting state functional connectivity in women with chronic migraine. Cephalalgia 38: 1731-1741.

14. Bingel U, Tracey I (2008) Imaging CNS modulation of pain in humans. Physiology 2008 23: 371-380.

15. Lorenz J, Minoshima S, Casey KL (2003) Keeping pain out of mind: The role of the dorsolateral prefrontal cortex in pain modulation. Brain 126: 1079-1091.

16. Apkarian AV, Bushnell MC, Treede RD, Zubieta JK (2005) Human brain mechanisms of pain perception and regulation in health and disease. Eur $\mathrm{J}$ Pain 9: 463-484

17. Ushida T, Fukumoto M, Binti C, Ikemoto T, Taniguchi S, et al. (2010) Alterations of contralateral thalamic perfusion in neuropathic pain. Open Neuroimag J 4:182-186.

18. Lundqvist C, Aaseth K, Grande RB, Benth JS, Russell MB (2010) The severity of dependence score correlates with medication overuse in persons with secondary chronic headaches: The Akershus study of chronic headache. Pain 148: 487-491.

19. Jahanshahi M, Obeso I, Rothwell JC, Obeso JA (2015) A fronto-striatosubthalamic-pallidal network for goal-directed and habitual inhibition. Nat Rev Neurosci 16: 719-732.

20. Haber SN (2003) The primate basal ganglia: Parallel and integrative networks. J Chem Neuroanat 26: 317-330.

21. Lüscher C, Malenka RC (2011) Drug-evoked synaptic plasticity in addiction From molecular changes to circuit remodeling. Neuron 69: 650-663.

22. Everitt BJ, Robbins TW (2005) Neural systems of reinforcement for drug addiction: From actions to habits to compulsion. Nat Neurosci 8: 1481-1489.

23. Aron AR, Robbins TW, Poldrack RA (2014) Right inferior frontal cortex: Addressing the rebuttals. Front Hum Neurosci 8: 905.

24. Glass JM, Williams DA, Fernandez-Sanchez ML, Kairys A, Barjola P, et al. (2011) Executive function in chronic pain patients and healthy controls: Different cortical activation during response inhibition in fibromyalgia. J Pain 12: $1219-1229$. 